\title{
AVALIAÇÃO DA EFICIÊNCIA DO MÉTODO TO-15 PARA DETERMINAÇÃO DE COMPOSTOS ORGÂNICOS VOLÁTEIS EM CONDIÇÕES TÍPICAS DE AMBIENTE URBANO
}

\author{
Cleyton Martins da Silva ${ }^{a, b}$, Elaine Cesar C. A. Souza ${ }^{b}$, Luane Lima da Silva ${ }^{\text {b }}$, Rafael Lopes Oliveira ${ }^{\text {b }}$, Graciela Arbilla ${ }^{\mathrm{b}}$ e \\ Sergio Machado Corrêa ${ }^{a, *}$ \\ aDepartamento de Química e Ambiental, Faculdade de Tecnologia, Universidade do Estado do Rio de Janeiro. 27537-000 Resende \\ - RJ, Brasil \\ bDepartamento de Físico-Química, Instituto de Química, Universidade Federal do Rio de Janeiro, 21949-900 Rio de Janeiro - RJ, \\ Brasil
}

Recebido em 16/05/2016; aceito em 15/06/2016; publicado na web em 05/08/2016

\begin{abstract}
EVALUATION OF TO-15 METHOD EFICIENCY TO DETERMINE VOLATILE ORGANIC COMPOUNDS IN TYPICAL URBAN CONDITIONS. The efficiency of U.S.EPA TO-15 Method was evaluated under typical conditions of an urban environment with natural and anthropic emission sources. The performance criteria which should be met for a system to qualify, were fulfilled: a detection limit of $\leq 0.2 \mathrm{ng}$ for all target compounds, replicate precision for a calibration standard and ambient samples within $20 \%$. The stability of the target compounds during storage of sampled air in canisters was also evaluated at atmospheric pressure and relative humidity of $50 \%$. Results show that, in the sampling conditions, compounds are not stable for more than a week. For validation experiments, samples were collected at the entrance of Tijuca National Park, located at the city of Rio de Janeiro. Isoprene was selected as a marker of biogenic emissions, and aromatic compounds were selected as markers of anthropogenic emissions, primarily vehicular emissions. Then, samples were collected in Saens Peña Square, a central area in the Tijuca District, in the northern part of the city, approximately $10 \mathrm{~km}$ from the entrance of Tijuca National Park. Volatile organic compounds were determined and the results were compared with previous data obtained in the same local using other methods.
\end{abstract}

Keywords: volatile organic compounds; isoprene; aromatic compounds; TO-15 method.

\section{INTRODUÇÃO}

São definidos como compostos orgânicos voláteis (COVs) os compostos orgânicos cuja pressão de vapor a $20{ }^{\circ} \mathrm{C}$ é inferior à pressão atmosférica normal $\left(1,013 \times 10^{5} \mathrm{~Pa}\right)$ e maior do que $130 \mathrm{~Pa}^{1}$ A legislação dos Estados Unidos ${ }^{2}$ lista ainda 189 COVs considerados potencialmente perigosos para a saúde humana. Com o objetivo de atender às exigências da legislação, a Agência Ambiental dos Estados Unidos (U.S.EPA) desenvolveu o método TO- $15,{ }^{3}$ que permite a determinação dos 97 principais compostos dessa lista. O método TO-15 estabelece os procedimentos gerais para a coleta de COVs usando canisters, botijões de aço inox eletropolidos internamente, e sua determinação utilizando a técnica de cromatografia gasosa acoplada à espectrometria de massas e termodessorção para injeção das amostras (CG-EM-DT). Estabelece também critérios de qualidade que devem ser atingidos na implementação do método: limite de detecção $\leq 0,5$ ppbv, precisão das replicatas dentro de $25 \%$ e exatidão dentro do $30 \%$ para as concentrações esperadas em amostras ambientais (0,5 a 25 ppbv).

Contudo, os resultados dependem de diversos fatores, tais como o processo de limpeza dos canisters, a umidade do ar coletado, o método de coleta, a estabilidade das amostras, as condições utilizadas na transferência das amostras e as condições cromatográficas, entre outras.

A estabilidade das amostras é um fator crítico. Sob as condições normalmente encontradas em amostras ambientais é geralmente aceito que as amostras podem ser guardadas por trinta dias. ${ }^{3-5}$ Entretanto, a estabilidade não é conhecida sobre condições variadas. Existe a possibilidade de perdas devidas à adsorção física dos compostos nas paredes do canister e dissolução dos compostos na água condensada nas paredes. Estes processos geralmente acontecem numa escala de

*e-mail: sergiomc@uerj.br tempo de minutos a horas e depende da temperatura, o material do revestimento interno do canister, a pressão e a umidade. Geralmente num prazo maior (dias ou semanas) podem acontecer perdas por reações químicas. Também podem acontecer perdas devidas a outros processos pouco conhecidos como degradação biológica.

Os COVs são provenientes tanto de fontes naturais quanto antrópicas. ${ }^{6-10}$ As plantas emitem grandes quantidades de COVs, isopreno (2-metil-1,3-butadieno), monoterpenos, sesquiterpenos e compostos orgânicos oxigenados. Dados da literatura mostram que o isopreno não é acumulado nas plantas e que as emissões dependem da quantidade de radiação e da temperatura, sendo maiores nas regiões tropicais e durante o verão. ${ }^{7,10}$ Os compostos de origem antrópica são devidos principalmente aos processos de queima de combustíveis fosseis e queima de biomassa. As emissões antrópicas acontecem principalmente nas regiões industrializadas (95\% no Hemisfério Norte com um pico entre $40^{\circ} \mathrm{N}$ e $50^{\circ} \mathrm{N}$ ), e nas grandes cidades devido ao trânsito de veículos. ${ }^{6-10}$

A cidade de Rio de Janeiro apresenta características peculiares. Com uma população de 6.476 .631 habitantes ${ }^{11}$ e uma frota veicular de 2.960 .380 veículos, ${ }^{12}$ as emissões veiculares são a maior fonte de poluentes. Ao mesmo tempo, a cidade abriga o Parque Nacional da Tijuca, que com $39,53 \mathrm{~km}^{2}$ é a maior floresta secundária urbana do mundo, com acesso pelas zonas sul, norte e oeste da cidade, ${ }^{13}$ e o Parque Estadual da Pedra Branca na zona oeste do município do Rio de Janeiro. ${ }^{14}$ Este último compreende todas as áreas situadas acima da linha de cota de $100 \mathrm{~m}$ do Maciço da Pedra Branca e seus contrafortes e, com uma área de $124,92 \mathrm{~km}^{2}$, é considerada a maior floresta urbana primária do mundo. Destas e de outras importantes áreas verdes da cidade, pode ser esperada uma contribuição importante das emissões biogênicas, principalmente isopreno.

Existem poucos estudos sobre as concentrações de compostos orgânicos nessas áreas verdes ${ }^{15,16}$ principalmente no referido às 
concentrações de isopreno. ${ }^{17}$ Esses estudos poderiam contribuir para um melhor conhecimento da composição do ar desta região e, da interação entre as áreas verdes e as áreas urbanizadas.

Já a maior parte das determinações de compostos orgânicos voláteis não oxigenados na cidade de Rio de Janeiro foi realizadas em áreas com predominância de emissões veiculares e tiveram como objetivo a determinação de compostos aromáticos usando o método de coleta com cartuchos adsorvedores ${ }^{18-21}$ ou a especiação de COVs para a realização de modelagens numéricas da qualidade do $\mathrm{ar}^{22,23}$ motivo pelo qual foram feitas poucas coletas de material. Num trabalho publicado em 2006, Alves et al. ${ }^{10}$ discutiram a importância das contribuições das fontes naturais, assim como diversas técnicas de coleta e análise de COVs, usando canisters e tubos de aço inox com Tenax TA, que foram aplicadas para um estudo numa área rural de Portugal.

O método TO-15 se mostra como um dos mais apropriados para estudos em áreas de floresta e/ou urbanizadas, por não requerer infraestrutura de apoio, tal como energia elétrica no local da coleta nem a utilização de bombas de ar, e por permitir, ainda, a amostragem durante períodos de tempo entre 1 minuto e 24 horas, cobrindo assim diversas necessidades. Não existem dados na literatura a respeito das incertezas sobre a reprodutibilidade do método, especialmente no caso de compostos muito reativos como o isopreno, e no caso de amostras coletadas em locais com alta umidade como é o ambiente perto da floresta e outras áreas verdes. Assim, o presente trabalho avalia o método TO-15 e suas várias modificações com respeito aos métodos discutidos previamente. ${ }^{10}$

O principal objetivo deste estudo é avaliar a eficiência do método TO-15 na determinação de compostos orgânicos voláteis em amostras coletadas no ambiente urbano do Rio de Janeiro, principalmente em regiões próximas à área verde da cidade. Para atingir este objetivo foram realizados diversos testes para verificar o desempenho do método e analisados os principais compostos orgânicos voláteis de origem antropogênica e o isopreno, que é o mais abundante composto orgânico volátil de origem biogênica. Finalmente, o método foi aplicado para determinar as concentrações dos compostos orgânicos voláteis coletados numa área urbana próxima à Floresta da Tijuca.

\section{PARTE EXPERIMENTAL}

\section{Descrição geral do método TO-15}

O método TO-15 foi desenvolvido e avaliado pela U.S.EPA. ${ }^{3}$ As amostras de ar são coletadas em canisters, botijões de aço inox tratados para garantir uma superfície inerte. Em geral os canisters são eletropolidos internamente e posteriormente submetidos a um tratamento químico para passivação. As amostras podem ser introduzidas nos canisters, previamente submetidos a vácuo, à pressão atmosférica ou menor, permitindo uma coleta passiva sem o uso de uma bomba auxiliar, ou a pressões maiores que a atmosférica para o qual é necessário um sistema auxiliar para pressurização. Neste trabalho se optou pela amostragem passiva e a introdução de amostra até atingir pressão atmosférica.

Após a coleta, a válvula do canister é fechada e o mesmo é transportado ao laboratório para análise. A análise é realizada transferindo um volume conhecido diretamente do canister através de um sistema concentrador multiadsorvente. A eliminação da água da amostra é realizada durante a etapa de concentração e depende da composição do multiadsorvente, do tempo de passagem do gás de arrastre e da temperatura, condições a serem detalhadas. Após a concentração da amostra e a eliminação da água, a amostra é dessorvida termicamente e concentrada na entrada da coluna para iniciar a etapa de separação cromatográfica. Para o método TO- $15^{3}$ a identificação e quantificação dos COV são realizadas por espectrometria de massas em modo SCAN ou monitoramento seletivo de íons (MSI).

As principais diferenças entre os métodos TO-15 e TO-14A residem no tratamento da água, no método de concentração da amostra e no tipo de detectores usados na determinação cromatográfica. ${ }^{3}$ No método TO-14A a eliminação da água é realizada usando uma membrana permeável Nafion ${ }^{\circledR}$ (baseada num copolímero de tetrafluoroetileno). A amostra passa por esta membrana antes de ser pré-concentrada. Por este motivo, compostos leves polares serão também retidos na membrana permeável e não poderão ser determinados. A concentração da amostra é realizada usando um tubo de níquel (geralmente de $0,32 \mathrm{~cm}$ de diâmetro interno) com esferas de vidro Pyrex ${ }^{\circledR}$ de 60-80 mesh, ou equivalente, resfriado com nitrogênio líquido. Posteriormente a amostra é aquecida (de $-160{ }^{\circ} \mathrm{C}$ a $120{ }^{\circ} \mathrm{C}$ em 60 segundos) e injetada no cromatógrafo. O método TO-14A contempla o uso de diversos detectores (espectrometria de massas, ionização de chama, fotoionização ou captura de elétrons). ${ }^{3,5}$

O método TO-15 fornece um sistema alternativo de tratamento da água, usando concentradores multiadsorventes a temperaturas próximas à ambiente, permitindo a purga seletiva da água e a posterior análise de compostos polares como álcoois, cetonas e ésteres. A concentração da amostra é feita num pequeno tubo contendo diversos multiadsorventes a temperaturas geralmente entre $-25{ }^{\circ} \mathrm{C}$ e $25{ }^{\circ} \mathrm{C}$, segundo o tratamento prévio que tenha sido feito com a água. Eventualmente, dependendo da configuração do aparelho, o leito adsorvedor pode ser resfriado com dióxido de carbono ou por um sistema Peltier, desde que a água seja retida previamente. Em alguns casos, nos quais não serão analisados compostos polares, a membrana permeável Nafion ${ }^{\circledR}$ é utilizada para eliminar a água. O método TO-15 contempla apenas o uso de espectrometria de massas como sistema de detecção e estabelece critérios mínimos de qualidade que devem ser atingidos.

$\mathrm{Na}$ Figura 1 é apresentado um esquema geral do método com as diversas etapas: limpeza dos canisters, coleta das amostras, transferência e análise. Os detalhes de cada um dos procedimentos são discutidos em continuação.

\section{Descrição dos canisters}

Um canister é um recipiente de aço inoxidável com uma válvula, também totalmente em aço inox, desenhada para conservar amostras no intervalo de pressões $10 \mathrm{~mm} \mathrm{Hg} \mathrm{a} 40$ psig. Existem canisters de diversos tamanhos, geralmente entre $400 \mathrm{~mL}$ e $6 \mathrm{~L}$ dependendo da utilização que será dada. Em geral, canisters menores são usados para amostras mais concentradas. O interior do canister deve ser inerte para evitar a degradação das amostras. Existem dois tipos de tratamento: os canisters SUMMA ${ }^{\circledR}$ ou TO-Can ${ }^{\circledR}$ são eletropolidos num procedimento no qual a parede interna é enriquecida em óxido de cromo-níquel (NiCrOx), protegendo a amostra do contato direto com o material do canister, que contem $70 \%$ de ferro, metal muito reativo e catalítico. O revestimento de $\mathrm{NiCrOx}(500-1000 \AA)$ evita a exposição ao ferro e evita o processo de corrosão devido ao ozônio, oxigênio, $\mathrm{NO}_{\mathrm{x}}$ e outros compostos oxidantes. Já os canisters SilcoCan ${ }^{\circledR}$ recebem um tratamento adicional (Silonite ${ }^{\circledR}$ ) com um revestimento cerâmico de aproximadamente $500 \AA$ que os torna inertes não apenas para os compostos orgânicos menos reativos, mas também para os compostos com enxofre.

Neste trabalho foram utilizados canisters de $6 \mathrm{~L}$, marca Restek, com tratamento Silonite ${ }^{\circledR}$ e válvula TOV-2 ${ }^{\mathrm{TM}}$.

\section{Limpeza dos canisters}

O procedimento básico consiste na realização de seis ciclos de limpeza, sendo que nos três primeiros é introduzida uma mistura 


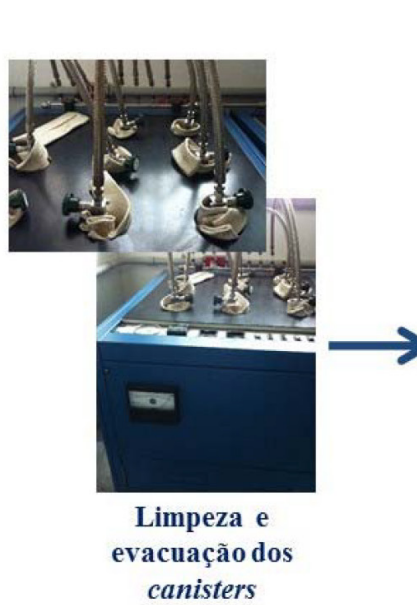



Coleta da amostra canisters


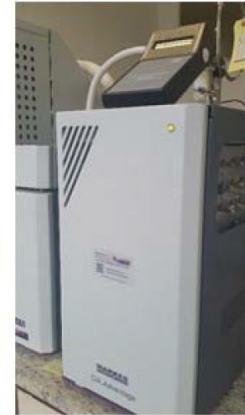

Transferência da amostra com o auxilio da unidade de termodessorção

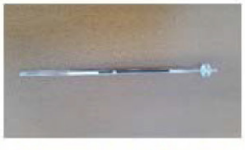

Adsorção $\left(-10^{\circ} \mathrm{C}\right) \mathrm{e}$ dessorção $\left(300^{\circ} \mathrm{C}\right)$ na Cold Trap

Figura 1. Esquema do Método TO-15 da U.S. EPA tal como implementado neste trabalho

He-vapor de água e nos três seguintes é introduzido He. A função da água no gás umidificado é hidrolisar as impurezas no canister e ocupar os sítios ativos na superfície interior, deslocando as impurezas e permitindo que sejam evacuadas. Durante cada ciclo, os canisters são despressurizados e no último ciclo é realizado vácuo abaixo de 0,05 $\mathrm{mm} \mathrm{Hg}$. Durante todo o processo de limpeza, a temperatura do sistema é mantida em $120^{\circ} \mathrm{C}$. Alternativamente, dependendo do equipamento utilizado, pode ser usado $\mathrm{N}_{2}$ ou ar sintético ultrapuros e, inclusive, realizar a limpeza só com ciclos secos. Neste trabalho foi utilizado um equipamento da empresa Teledyne Hasting Instruments, modelo Omega CN9000A e realizados, em média, quatro ciclos de limpeza usando uma mistura He-vapor de água e, posteriormente, 4 ciclos com He seco para atingir os critérios de qualidade desejados.

O controle do procedimento de limpeza deve ser realizado inicialmente em todos os canisters. Após a limpeza, os canisters são pressurizados com $\mathrm{He}$ ou ar ultrapuro e analisados por CG-EM. Conforme as recomendações do método $\mathrm{TO}-15,{ }^{3}$ o processo de limpeza é considerado eficiente se a concentração dos compostos de interesse é menor que 0,2 ppbv. Nesse caso os canisters são evacuados novamente $(<0,05 \mathrm{~mm} \mathrm{Hg})$ e guardados até sua utilização, caso contrário, deverão ser submetidos a um novo procedimento de limpeza. Após certificado o funcionamento apropriado do sistema de amostragem, o procedimento de controle pode, eventualmente, ser reduzido para um número menor de canisters (brancos). Para canisters novos, que não foram utilizados previamente, é geralmente necessário fazer um maior número de ciclos de limpeza até atingir os critérios de qualidade estabelecidos, eliminando compostos adsorvidos nas paredes e que presumivelmente foram utilizados no processo de fabricação.

A fim de evitar a adsorção de amostra residual ou a entrada de ar ambiente através da válvula, é recomendável que canisters que serão armazenados durante algum tempo sejam previamente submetidos a dois ciclos de limpeza com He-vapor de água e guardados com $\mathrm{He}$ no seu interior até serem utilizados novamente. A limpeza completa, tal como descrita, é realizada preferencialmente no dia anterior à coleta de amostra.

\section{Coleta das amostras}

Como já mencionado, as amostras de ar podem ser coletadas de duas formas: coleta passiva, a pressão atmosférica ou menor, e coleta a pressão maior que a atmosférica usando uma bomba de ar. Neste trabalho foi realizada a coleta passiva à pressão atmosférica. $\mathrm{O}$ método TO-15 contempla duas formas de introduzir o ar atmosférico no canister previamente evacuado: coleta instantânea, na qual a válvula é aberta até igualar a pressão interna e externa (normalmente de $10 \mathrm{a}$ 30 segundos) e coleta com um restritor de fluxo durante um período determinado e em condições controladas. Normalmente, usando este tipo de válvulas, a velocidade de entrada do gás no canister diminui na medida em que a pressão interna aumenta. O uso do restritor de fluxo permite que a entrada da amostra seja realizada de forma controlada. Neste trabalho foram usados ambos os procedimentos para comparação. O controle da entrada do ar para dentro do canister foi realizado usando um restritor com uma válvula Restek com um fluxo de 67-83 $\mathrm{mL} \mathrm{min}^{-1}$ calibrado de forma que, utilizando um canister de $6 \mathrm{~L}$, fosse atingida a pressão final de $1 \mathrm{~atm}$ em uma hora de coleta (Passive Canister Sampler CS1200E com um filtro de Silonite®).

\section{Análise por cromatografia}

As amostras de ar foram analisadas utilizando a técnica de cromatografia gasosa/espectrometria de massas, utilizando termodessorção para injeção das amostras (DT-CG-EM).

Inicialmente, a transferência das amostras para o cromatógrafo foi realizada através da unidade amostradora de canisters CIA Advanced da Markes. Um volume pré-determinado (100 a $500 \mathrm{~mL}$ ) de amostra gasosa foi transferido do canister e passou através de um loop de peneira molecular a fim de reter a umidade $\left(\mathrm{Nafion}^{\circledR}\right)$. Em seguida, 
os COVs foram retidos em uma armadilha fria (cold trap) contendo 3 leitos (Air Toxics Analyser contendo Tenax TA, Carbograph 1TD e Carboxen 1003) dentro da unidade de termodessorção a uma temperatura de $-10{ }^{\circ} \mathrm{C}$ e posteriormente dessorvidos a $300{ }^{\circ} \mathrm{C}$. Em seguida, os analitos foram concentrados na entrada da coluna para iniciar o processo de separação cromatográfica.

As amostras foram analisadas em um cromatógrafo a gás Agilent 7890A acoplado a um espectrômetro de massas Agilent 5975C. A separação dos COVs foi realizada utilizando uma coluna capilar DB-624, com $60 \mathrm{~m}$ de comprimento, 0,32 mm de diâmetro interno e 1,8 $\mu \mathrm{m}$ de espessura de filme. A DB-624 é composta de $6 \%$ de cianopropil-fenil e $9 \%$ de dimetilsiloxano e possui média polaridade, podendo ser utilizada no intervalo de temperatura de $40{ }^{\circ} \mathrm{C}$ a $260{ }^{\circ} \mathrm{C}$.

Os parâmetros utilizados na etapa de injeção das amostras foram: temperatura de adsorção (traplow): $-10{ }^{\circ} \mathrm{C}$; temperatura de dessorção (trap high): $300{ }^{\circ} \mathrm{C}$; temperatura da linha de transferência: $150{ }^{\circ} \mathrm{C}$; tempo de purga da linha: 1 minuto; vazão de purga da linha: $20 \mathrm{~mL}$ $\min ^{-1}$; vazão de purga do trap: $50 \mathrm{~mL} \mathrm{~min}^{-1}$; injeção (em modo split):

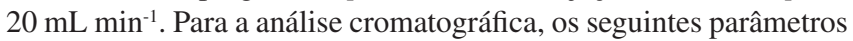
foram utilizados: temperatura inicial do forno: $25^{\circ} \mathrm{C}$; isoterma: 2 minutos; rampa de $25{ }^{\circ} \mathrm{C}$ a $50{ }^{\circ} \mathrm{C}: 0,8{ }^{\circ} \mathrm{C} \mathrm{min}^{-1}$; rampa de $50{ }^{\circ} \mathrm{C}$ a $250{ }^{\circ} \mathrm{C}: 5{ }^{\circ} \mathrm{C}$ minn $^{-1}$; post run: 3 minutos a $250{ }^{\circ} \mathrm{C}$; pressão: 20 psi (constante) sem divisão de fluxo; temperatura da linha de transferência para o detector: $250{ }^{\circ} \mathrm{C}$. $\mathrm{O}$ espectrômetro de massas operou a $70 \mathrm{eV}$ em modo seletivo de íons.

\section{Curvas e parâmetros analíticos}

Para a obtenção das curvas analíticas, utilizou-se o método da padronização externa (com superposição de matriz), pela comparação da área do pico cromatográfico do COV a ser quantificado na amostra com as áreas obtidas utilizando padrões de concentrações conhecidas. Esta comparação é realizada por uma equação da reta, com respectivo coeficiente de determinação. Um valor de coeficiente de determinação de 0,999 é considerado ótimo, ${ }^{24}$ entretanto a ANVISA ${ }^{25}$ sugere um valor de 0,98 , valor que coincide com o recomendado por outros autores. ${ }^{26}$

O método TO-15 descreve o procedimento para preparo de padrões de trabalho (working standards) pela técnica de diluição dinâmica, que requer um sistema complexo de manipulação de gases e controladores de vazão mássicos (massflow). A alternativa utilizada neste trabalho é injetar no cromatógrafo diferentes volumes do padrão gasoso, utilizando o próprio controlador de vazão mássico equipamento. $\mathrm{O}$ instrumento determina o valor da massa de gás por unidade de tempo e este valor depende da densidade do gás, que por sua vez depende da temperatura, pressão e composição do gás. É importante salientar que o padrão certificado deverá estar em uma concentração próxima à da amostra, tal que seja possível obter a curva analítica com precisão.

A identificação dos analitos foi realizada preliminarmente com auxílio do espectro de massas e, posteriormente, as curvas analíticas foram obtidas utilizando padrão gasoso certificado (Linde Spectra Restek), contendo 57 componentes. A fim de cobrir todo o intervalo de concentrações foram usados dois padrões: Restek 34445 (Padrão 1, Padrão de calibração de gases para monitoramento do ar, percursor de Ozônio/PAMS Mix, com concentrações na faixa 20-60 ppbC em nitrogênio) e Restek 26369 (Padrão 2, Padrão de calibração de gases para monitoramento do ar, percursor de Ozônio/PAMS Mix, com concentrações de 100 ppb em nitrogênio).

Diferentes volumes foram injetados para obtenção da curva analítica e, uma vez que o gás contido no padrão foi submetido às condições de adsorção, foi possível determinar a massa de cada analito de interesse que ficou retida na armadilha fria. $\mathrm{O}$ software do equipamento forneceu o valor exato de cada volume injetado através do medidor de vazão mássico. Utilizando cada valor de volume injetado e as respectivas concentrações fornecidas pelo certificado de análise do padrão, foi possível determinar a massa (em ng) injetada de cada analito de interesse e obter as curvas analíticas. A fim de considerar todas as concentrações possíveis nos ambientes estudados, foram construídas duas curvas, uma em um intervalo de concentrações menores, com o padrão de concentrações 20-60 ppbC (Padrão 1), e outra em um intervalo de concentrações maiores, com o padrão de 100 ppb (Padrão 2). Cada curva analítica foi construída com 10 pontos em triplicata, sendo injetados para os dois padrões volumes na faixa 10 a $350 \mathrm{~mL}$. Para todos os compostos as curvas analíticas tiveram $\mathrm{R}^{2} \geq 0,99$ e apresentaram linearidade no intervalo de trabalho. As faixas de massa para cada composto variaram, já que as concentrações dos compostos nos padrões são diferentes. A modo de exemplo, o intervalo da curva para o benzeno foi de 0,37 a 7,44 (Padrão 1) e 5,10 a 63,80 $\mu \mathrm{g} \mathrm{m}^{-3}$ (Padrão 2) e os coeficientes de determinação de 0,996 para ambas as curvas. Para o tolueno os intervalos de concentração foram de 0,50 a 10,10 (Padrão 1) e 7,22 a 90,30 $\mathrm{gg} \mathrm{m}^{-3}$ (Padrão 2) e os coeficientes de correlação 0,991 e 0,997, respectivamente.

Diariamente foram realizados brancos do sistema de injeção e conferido um ponto da curva de calibração $(100 \mathrm{~mL})$, conforme recomendado nas especificações do método TO- 15 . $^{3}$

No Material Suplementar é mostrado um cromatograma do branco (Figura 1S), dos compostos de contidos no padrão Restek, obtido em modo SCAN (Figura 2S) e de uma amostra típica, obtido em modo monitoramento seletivo de íons (Figura 3S). Na Tabela 1S são listados os COVs analisados e os íons utilizados para a quantificação e as massas moleculares correspondentes. Para o procedimento de controle de qualidade foram analisados os seguintes compostos: propeno, buteno, isopreno, hexano, benzeno, tolueno, etil benzeno, $\mathrm{m}+\mathrm{p}$-xilenos e o-xileno.

Limite de detecção (LD): é a menor quantidade de substância que pode ser detectada, mesmo que não necessariamente possa ser quantificada. ${ }^{24}$ Os dois métodos mais usados para sua determinação são o baseado na relação sinal-ruído e o baseado nos parâmetros da curva analítica. ${ }^{24}$ No primeiro caso é realizada a comparação entre a medição dos sinais de amostras em baixas concentrações conhecidas do composto de interesse na matriz e um branco (matriz isenta do composto de interesse) destas amostras. Neste trabalho foi admitido que uma relação sinal-ruído de 3:1 era aceitável como LD. Posteriormente, usando os parâmetros da curva analítica construída com valores de concentração de padrão próximos ao LD determinado anteriormente, foi calculado um valor como:

$$
L D=3,3 \frac{s}{S}
$$

em que s é a estimativa do desvio padrão do coeficiente linear da equação e $S$ é a inclinação ou coeficiente angular da curva analítica. ${ }^{24}$

O LD foi determinado em unidades de massa do composto de interesse e, assim, para uma dada concentração esperada do composto no ar atmosférico que está sendo estudado, foi estimado o volume mínimo de ar (na realidade massa de ar) que deveria ser injetado no CG.

Limite de quantificação $(L Q)$ : é a mínima quantidade que pode ser medida para o procedimento experimental que está sendo utilizado. ${ }^{24}$ Os mesmos critérios de LD podem ser adotados para o LQ, utilizando a relação:

$$
L D=10 \frac{S}{S}
$$

Repetitividade: representa a concordância entre os resultados de medições sucessivas de um mesmo método, efetuadas sob as mesmas condições de medição, chamadas condições de repetitividade: mesmo 
procedimento; mesmo analista; mesmo instrumento usado sob as mesmas condições; mesmo local; repetições em um curto intervalo de tempo. Neste caso foi determinada passando iguais volumes do padrão certificado. ${ }^{24}$ Neste trabalho foram realizadas 10 determinações de uma quantidade de padrão predeterminada $(50 \mathrm{~mL}$ do padrão 2$)$. Conforme recomendação do método TO- $15,{ }^{3}$ a precisão das replicatas deve ser calculada como:

$$
P R=100 \frac{\left|x_{1}-x_{2}\right|}{x_{m}}
$$

em que $\mathrm{x}_{1}$ e $\mathrm{x}_{2}$ são os valores obtidos para duas determinações e $\mathrm{x}_{\mathrm{m}}$ é o valor médio. Neste trabalho foi usada a equação:

$$
P R=100 \frac{\left|x_{\max }-x_{\min }\right|}{x_{m}}
$$

em que $\mathrm{x}_{\max }$ e $\mathrm{x}_{\min }$ são o maior e o menor valores, respectivamente obtidos para as dez determinações e $\mathrm{x}_{\mathrm{m}}$ é o valor médio.

\section{Avaliação da qualidade das coletas}

A fim de verificar a qualidade do método de coleta, foram realizadas as seguintes determinações: determinação da estabilidade da amostra guardada no canister, determinação da repetitividade de replicatas verdadeiras e de sucessivas determinações cromatográficas da mesma amostra, avaliação do uso de restritores de fluxo em comparação a coletas instantâneas.

Estabilidade das amostras: A estabilidade das amostras nos canisters foi certificada realizando coletas simultâneas de ar atmosférico em condições reais. Foi escolhido um local de coleta com condições críticas de umidade e composição, com contribuição de fontes biogênicas e antrópicas. Foram coletadas 3 amostras simultâneas usando os canisters de $6 \mathrm{~L}$ e analisadas no dia seguinte a coleta, 15 e 30 dias depois. Foram analisados os seguintes compostos: propeno, buteno, isopreno, hexano, benzeno, tolueno, etil benzeno, $\mathrm{m}+\mathrm{p}$-xilenos e o-xileno.

Repetitividade dos resultados para coletas simultâneas: Foram feitas duas coletas simultâneas, com os canisters de 6 L, usando um restritor de fluxo de 1 hora.

Comparação das coletas com e sem restritor de fluxo: Foram comparados os resultados obtidos coletando as amostras usando um restritor de uma hora (duas coletas) e coletas instantâneas (abertura da válvula do canister previamente evacuado que permite a entrada do gás de 30 a 60 segundos).

\section{Local de coleta das amostras}

As amostras utilizadas para a avaliação do método foram coletadas na Praça Afonso Viseu, no Alto da Boa Vista, Rio de Janeiro (coordenadas -22 $57^{\prime} 40,43^{\prime \prime}$ S e -43 $16^{\circ} 24,20^{\prime \prime} \mathrm{W}$ ). O local, na entrada do setor Floresta da Tijuca do Parque Nacional da Tijuca, é arborizado, com construções residenciais, fluxo moderado de pessoas e veículos e a circulação de duas linhas de ônibus que unem a Rodoviária Novo Rio e a Barra da Tijuca. Abriga um restaurante de médio porte, o chafariz do francês Grandjean de Montigny e é ponto de encontro de grupos de caminhada e lugar de recreação de crianças e adultos. Em torno da praça, visitantes do parque frequentemente estacionam veículos. O local foi escolhido porque está sujeito a emissões naturais e antropogênicas e apresenta condições ambientais típicas da área urbana cercada pela Mata Atlântica. Nos dias de coleta a temperatura ambiente foi de 25 a $27^{\circ} \mathrm{C}$ e a umidade do ar em torno de $50 \%$. Todas as amostras foram coletadas entre as 11 e 13 horas em dias úteis, nos quais o movimento de pessoas na praça é reduzido em comparação com dias de final de semana e feriados.
Para ilustrar a aplicabilidade do método, foram coletadas, também, outras amostras na Praça Saens Peña, no bairro da Tijuca da Cidade de Rio de Janeiro (22 $55^{\prime} 29.9^{\prime \prime}$ 'S 4313'57.2”W). A região se encontra aproximadamente a $10 \mathrm{~km}$ de uma das entradas do Parque Nacional da Tijuca e a menos de $1 \mathrm{~km}$ do Morro de Sumaré, com 700 $m$ de altitude e vegetação da mata Atlântica. O local possui grande número de estabelecimentos comerciais e intenso fluxo de veículos, principalmente veículos leves, assim como uma estação de metrô. As concentrações de COVs nesse local já tinham sido estudadas previamente usando cartuchos adsorvedores ${ }^{16,18} \mathrm{e}$ o método TO-14. $\mathrm{A}^{23}$ e, por isto, esse lugar de coleta parece apropriado para avaliar a aplicabilidade do método. Durante o período de coleta a temperatura foi de 26 a $32,9{ }^{\circ} \mathrm{C}$ e a umidade do ar em torno de $65 \%$.

\section{RESULTADOS E DISCUSSÃO}

\section{Limites de detecção e quantificação e precisão das replicatas}

Inicialmente a seletividade do método foi avaliada para garantir que o pico de resposta dos analitos, no tempo de retenção característico, fosse proveniente exclusivamente do mesmo.

Os valores de LD determinados neste trabalho se encontraram no intervalo 0,1 a 0,2 ng. Isso significa que sendo injetados volumes de $500 \mathrm{~mL}$ de ar, como geralmente recomendado, ${ }^{3}$ será possível detectar compostos que estão em concentrações de aproximadamente 0,2-0,4 $\mathrm{mg} \mathrm{m}^{-3}$. Esses valores estão num bom acordo com o LD indicado para o método TO-15 de 0,2 ppbv. ${ }^{3}$

A precisão determinada para a análise de 10 amostras, em condições de concentração próxima a que encontrada nas amostras, como já descrito, foi estimada usando a Equação 4, dando um desvio $\leq$ $20 \%$. Esses valores são afetados por diversos fatores, como massa molecular, solubilidade em água, polarizabilidade etc., sendo que, em geral, a precisão é maior para compostos não polares. ${ }^{3}$ Os valores achados estão em bom acordo com os reportados no método TO-15 (valor típico $\leq 25 \%)^{3}$ e podem ser considerados razoáveis dado que envolvem todos o procedimento de transferência (determinação do volume de amostra gasosa, adsorção e dessorção na armadilha fria) e análise da amostra.

A exatidão dos resultados não pode ser verificada porque não existe material de referência certificado para este método. Esse problema poderá ser contornado no futuro comparando os resultados do método TO-15 com os de outro método ou fazendo calibração interlaboratorial.

\section{Avaliação da qualidade das coletas}

Estabilidade das amostras: A estabilidade das amostras depende de diversos fatores como material do canister, pressão e umidade da amostra, solubilidade em água e reatividade dos compostos. Por esse motivo, neste trabalho as amostras foram coletadas em condições próximas às esperadas no meio ambiente (pressão atmosférica, 50\% de umidade e $27^{\circ} \mathrm{C}$ ). De uma forma geral, o método TO- $15^{3,4}$ e os diversos fabricantes de canisters indicam que as amostras sejam analisadas em até 30 dias, mas recomendam que este período seja verificado para as condições específicas de cada estudo e considerando compostos de diversas reatividades. Neste trabalho foram escolhidos compostos muito reativos (isopreno, propeno e buteno), compostos aromáticos e o hexano que apresenta baixa reatividade em condições atmosféricas. Os resultados são mostrados na Tabela 1, na qual foram representados os valores de PR calculados como:

$$
P R=100 \frac{\left|x_{1}-x_{n}\right|}{x_{1}}
$$


em que $\mathrm{x}_{1}$ é o sinal analítico para a análise ao dia seguinte da coleta, $\mathrm{x}_{\mathrm{n}}$ é o sinal analítico para a amostra considerada (dia 15 ou 30). Todas as amostras foram analisadas em duplicata.

Tabela 1. Valores de PR (\%), calculados usando a equação 5, mostrando o desvio das áreas para as análises realizadas 15 e 30 dias depois, com respeito à análise realizada ao dia seguinte da coleta

\begin{tabular}{lcc}
\hline Composto & PR\% (Dia 15) & PR\% (Dia 30) \\
\hline Propeno & 1,94 & 80,98 \\
1-Buteno & 9,94 & 56,41 \\
Isopreno & 61,64 & 82,07 \\
Hexano & 191,19 & 92,85 \\
Benzeno & 60,24 & 91,12 \\
Tolueno & 37,53 & 72,15 \\
Etil benzeno & 44,60 & 64,21 \\
m+p-Xileno & 39,64 & 68,42 \\
o-Xileno & 48,93 & 69,36 \\
\hline
\end{tabular}

Os valores de PR são sempre apresentados como valores absolutos, mas podem acontecer desvios negativos ou positivos. ${ }^{20}$ Neste trabalho os desvios foram negativos para todos os compostos exceto para o hexano. Os desvios negativos são mais frequentes e geralmente são devidos à adsorção na parede do canister, reações químicas com água ou outros compostos presentes. ${ }^{4}$ Desvios positivos podem ser atribuídos à partição dos COVs entre a fase gás e a água condensada na superfície do canister, que depende da temperatura, pressão e umidade.

Análises sucessivas levam à diminuição de pressão, que reduz a quantidade de água condensada nas paredes e, portanto, aumenta a fração de composto orgânico na fase gás. ${ }^{20}$ Os valores de PR obtidos neste trabalho, para as condições de coleta, são, na maior parte dos casos, maiores que 25 , indicando que as amostras não são estáveis para esses períodos de estocagem. É importante mencionar que os estudos de estabilidade são geralmente realizados com amostras de padrão gasoso. Como discutido na literatura, ${ }^{27}$ amostras ambientais são muito mais complexas, já que contêm água, ozônio, compostos oxigenados, nitrogenados e compostos de enxofre que, além de reagir, podem interferir no processo de coleta, armazenamento e análise. Assim, é esperado que a estabilidade de amostras ambientais seja menor que a informada na literatura para amostras preparadas através da diluição de um padrão.

Os resultados para os compostos de maior interesse neste trabalho, isopreno e compostos aromáticos, mostram que a mudança na concentração é de aproximadamente $2-3 \%$ por dia.

Repetitividade dos resultados para coletas simultâneas: Foram calculados os valores de PR para as duas coletas simultâneas, com os canisters de $6 \mathrm{~L}$, usando um restritor de fluxo de 1 hora. Os valores calculados para a primeira análise das amostras, usando a Equação 3 , foram $6 \%, 19 \%, 21 \%, 22 \%, 20 \%, 21 \%, 14 \%, 15 \%$ e $19 \%$ para propeno, buteno, isopreno, hexano, benzeno, tolueno, etil benzeno, $\mathrm{m}+\mathrm{p}$-xilenos e o-xileno, respectivamente. Posteriormente foram realizadas análises sucessivas (replicatas, até um total de quatro injeções) de cada canister. Os resultados são mostrados na Tabela 2 onde foram representados os valores de PR calculados como:

$$
P R=100 \frac{\left|x_{\text {ninjeção }}-x_{1^{a} \text { injeção }}\right|}{x_{m}}
$$

em que $x_{n \text { injeção }}$ ó sinal analítico para a enésima injeção de cada um dos canisters, e $\mathrm{x}_{1}{ }^{\circ}$ injeção $\mathrm{e}$ o sinal analítico da primeira injeção do mesmo canister e $\mathrm{x}_{\mathrm{m}}$ é o valor médio de ambos os valores. Assim, este valor representa o desvio das sucessivas replicatas devido não apenas ao erro da determinação cromatográfica, mas também, às diferenças inerentes as injeções sucessivas devidas à mudança de pressão residual dentro do canister, que é agravada a cada injeção e a processos de adsorção/dessorção dos compostos.

Tabela 2. Valores de PR (\%) mostrando o desvio das áreas para as quatro replicatas de cada canister, calculados usando a Equação 6

\begin{tabular}{lccc}
\hline Composto & $2^{\text {a }}$ injeção & 3 $^{\text {a }}$ injeção & $4^{\text {a }}$ injeção \\
\hline Canister 1 & & & \\
\hline propeno & 9,9 & 18,8 & 19,9 \\
1-buteno & 6,3 & 11,9 & 16,4 \\
isopreno & 7,2 & 10,6 & 16,1 \\
hexano & 5,8 & 12,1 & 19,3 \\
benzeno & 2,6 & 9,5 & 13,0 \\
tolueno & 1,8 & 6,7 & 10,3 \\
etil benzeno & 3,6 & 2,6 & 3,8 \\
m+p-xileno & 4,4 & 6,4 & 5,7 \\
o-xileno & 3,5 & 5,1 & 8,6 \\
\hline Canister 2 & & & \\
propeno & 19,8 & 10,1 & 14,7 \\
1-buteno & 3,6 & 7,6 & 7,3 \\
isopreno & 2,3 & 9,1 & 11,0 \\
hexano & 1,2 & 6,2 & 8,0 \\
benzeno & 0,2 & 2,1 & 7,1 \\
tolueno & 1,6 & 5,1 & 6,0 \\
etil benzeno & 2,0 & 1,1 & 3,1 \\
m+p-xileno & $<0,1$ & 8,3 & 3,8 \\
o-xileno & 6,5 & 7,0 & 7,0 \\
\hline
\end{tabular}

Os resultados da Tabela 2 mostram que as análises sucessivas do mesmo canister, nas condições que foram utilizadas neste trabalho, são reproduzíveis dentro de uma incerteza de aproximadamente $\leq 20 \%$, que satisfaz o requerimento do método TO- 15 de $25 \%$. $^{3}$ Note-se que foram injetados $500 \mathrm{~mL}$ de gás em cada injeção, correspondendo para a quarta injeção a uma diminuição de pressão de aproximadamente 0,66 . O volume de $500 \mathrm{~mL}$ é geralmente apropriado para amostras ambientais, fornecendo sinais acima do LQ para os compostos de interesse e permitindo, caso sejam usados canisters de $6 \mathrm{~L}$, a realização de triplicatas com um valor de PR dentro dos critérios de qualidade.

Testes realizados no laboratório com canisters de menor capacidade $(1,5 \mathrm{~L})$ não se mostraram satisfatórios para a realização de triplicatas na análise de amostras ambientais em baixa concentração, provavelmente pela diminuição de pressão. Porém seriam apropriados para a análise de amostras provenientes de emissões veiculares, túneis e ambientes internos, com concentrações próximas as do Padrão 2 (100 ppb) para as quais seria possível injetar volumes menores de amostra.

Comparação das coletas com e sem restritor de fluxo: Como já mencionado para duas coletas simultâneas usando restritores de fluxo $(1 \mathrm{~h})$, os valores de PR calculados para a primeira análise das amostras, usando a Equação 3, foram 6\%, 19\%, 21\%, 22\%, 20\%, 21\%, $14 \%, 15 \%$ e $19 \%$ para propeno, buteno, isopreno, hexano, benzeno, tolueno, etil benzeno, $\mathrm{m}+\mathrm{p}$-xilenos e o-xileno, respectivamente, o que indica uma reprodutibilidade aceitável em comparação com os critérios de qualidade estabelecidos para o método TO-15. ${ }^{3}$

Na Tabela 3 são comparados os resultados obtidos coletando as 
amostras usando um restritor de uma hora (duas coletas) com coletas instantâneas (abertura da válvula do canister previamente evacuado que permite a entrada do gás de 30 a 60 segundos). As coletas instantâneas foram realizadas durante a coleta de 1 hora com restritor (nos minutos $0,15,30,45$ e 60). A comparação foi feita para a primeira injeção usando a relação da Equação 7:

$$
\text { Relação }=\frac{x_{\text {canistern }}}{x_{\text {média restritor }}}
$$

em que $\mathrm{x}_{\text {canister n }}$ é o sinal analítico para a primeira injeção de cada um dos canisters (coleta instantânea), e $\mathrm{x}_{\text {média restritor }}$ é a média do sinal analítico da primeira injeção dos dois canisters para os quais foi usado restritor de fluxo.

Como pode ser observada na Tabela 3, a maioria dos valores obtidos para as coletas instantâneas são maiores (em alguns casos num fator 2-3) que os valores obtidos para a coleta com restritor de fluxo. Assim, os valores médios para as coletas instantâneas (a média das cinco coletas) são significativamente maiores que o valor para a coleta de 1 hora, apresentando valores de PR de até $190 \%$. Os motivos dessa diferença podem estar relacionados a diversos fatores, tais como variações instantâneas das concentrações e, principalmente, fatores relacionados à abertura da válvula do canister, que determinam que o processo aconteça num tempo variável, geralmente 10 a 30 segundos. Contudo o método de coleta instantânea é considerado apropriado pela US-EPA ${ }^{3}$ e bastante prático em condições extremas de coleta, por exemplo, em locais de difícil acesso ou com temperaturas extremas, como é o caso da Antártica, e, portanto, seria necessário realizar outros experimentos no futuro para elucidar esta questão, em especial com amostragens em atmosferas de concentração conhecidas.

Tabela 3. Relações calculadas usando a Equação 7 entre as áreas concentrações obtidas para coletas instantâneas e coleta com restritor de fluxo

\begin{tabular}{lccccc}
\hline Composto & Canister 1 & Canister 2 & Canister 3 & Canister 4 & Canister 5 \\
\hline Propeno & 2,07 & 1,09 & 1,26 & 1,01 & 1,15 \\
1-Buteno & 2,03 & 1,09 & 1,26 & 1,01 & 1,15 \\
Isopreno & 2,03 & 1,07 & 1,38 & 1,57 & 1,72 \\
Hexano & 2,35 & 0,95 & 1,44 & 1,91 & 1,32 \\
Benzeno & 2,56 & 1,00 & 1,52 & 1,95 & 1,31 \\
Tolueno & 2,59 & 0,96 & 1,53 & 2,03 & 1,36 \\
Etil benzeno & 2,80 & 0,88 & 1,57 & 2,01 & 1,63 \\
m+p-Xilenos & 2,65 & 0,90 & 1,56 & 2,04 & 1,59 \\
o-Xileno & 2,90 & 0,78 & 1,67 & 2,00 & 1,79 \\
\hline
\end{tabular}

De uma forma geral, os resultados mostrados neste trabalho indicam que o método, tal como implementado, satisfaz os critérios de qualidade estabelecidos pela U.S.EPA, sendo recomendado para amostras ambientais o uso de canisters de $6 \mathrm{~L}$ e restritor de fluxo para controle da entrada da amostra, assim como a análise cromatográfica no prazo máximo de 3 a 4 dias. Finalmente, indicam que, como acontece em geral na determinação de concentrações de amostras ambientais, os resultados podem ser rigorosamente comparados apenas como outros obtidos nas mesmas condições de coleta e análise já que dependem de diversos fatores inerentes ao método e, também, das condições ambientais, como temperatura, umidade e composição da mistura.

O método de coleta com canisters e análise por cromatografia gasosa com detecção por ionização em chama ou espectrometria de massas tem sido avaliado por outros autores em diversas condições. Em alguns desses trabalhos ${ }^{10,28,29}$ foi comparado o desempenho da coleta com tubos de adsorção e com canisters. No trabalho desenvolvido por Colón et al. ${ }^{28}$ usando o método TO-14A e cartuchos de adsorção, os autores encontram uma precisão entre amostras coletadas com canisters em duplicatas, para compostos orgânicos voláteis não oxigenados, de 3 a 22\%, em bom acordo com os valores obtidos neste estudo. Em geral os autores consideram o método com canisters mais eficiente, apesar de ser mais caro e mais difícil de implementar. Por outro lado, como já mencionado, a coleta com canisters não requer energia elétrica nem equipamento adicional e pode ser realizada em diversos períodos de tempo.

\section{Aplicação do método}

O método foi aplicado a amostras coletadas na Praça Saens Peña no período 02 a 16 de março de 2015 . Foram coletadas 8 amostras, usando canisters de $6 \mathrm{~L}$ com restritor de fluxo de 1 hora entre as $8 \mathrm{e}$ 9 horas. Foram determinados 51 compostos $\left(\mathrm{C}_{4}-\mathrm{C}_{12}\right)$. Cada amostra foi analisada por triplicata injetando $500 \mathrm{~mL}$ e usando as condições já descritas. Todas as amostras foram analisadas em um período de, no máximo, três dias após a coleta. A curva de calibração e amostras de branco foram verificadas diariamente para garantir a qualidade das determinações. Os resultados (valores médios e desvio padrão) obtidos para os 25 compostos mais abundantes são mostrados na Tabela 4, assim como valores obtidos previamente neste local, em outros trabalhos, usando o método TO-14A e cartuchos ou tubos adsorvedores (método TO-2 da US.EPA).

Tabela 4. Valores médios de concentração $\left(\mathrm{em} \mathrm{mg} \mathrm{m}^{-3}\right.$ ) e desvio padrão ( $s d$ ) para os COVs coletados na Praça Saens Peña, no período 02 a 16 de março de 2015, determinados usando o Método TO-15. Valores de literatura (em $\mathrm{mg} \mathrm{m}^{-3}$ ) são apresentados para comparação

\begin{tabular}{|c|c|c|c|c|c|}
\hline & \multicolumn{2}{|c|}{$\begin{array}{c}\text { TO-15 (Este Trabalho) } \\
\text { canisters } \\
\end{array}$} & \multirow{2}{*}{$\begin{array}{l}\text { TO-14A }{ }^{23} \\
\text { canisters }\end{array}$} & \multirow{2}{*}{$\begin{array}{c}\text { TO-2 } \\
\text { cartuchos }\end{array}$} & \multirow{2}{*}{$\begin{array}{c}\text { TO-2 }{ }^{18} \\
\text { cartuchos }\end{array}$} \\
\hline & Média & $s d$ & & & \\
\hline n-butano & 22,9 & 11,1 & <L.D. & N.D. & N.D. \\
\hline isopentano & 14,2 & 7,8 & 19,3 & N.D. & N.D. \\
\hline n-pentano & 13,6 & 6,6 & N.D. & N.D. & N.D. \\
\hline isobutano & 12,5 & 5,5 & 20,5 & N.D. & N.D. \\
\hline 1-buteno & 9,0 & 5,2 & 2,9 & N.D. & N.D. \\
\hline tolueno & 7,5 & 3,3 & 14,9 & 4,8 & 7,02 \\
\hline n-heptano & 6,6 & 5,6 & 5,1 & N.D. & N.D. \\
\hline n-hexano & 5,6 & 2,8 & 5,5 & N.D. & N.D. \\
\hline isopreno & 4,3 & 3,4 & N.D. & N.D. & N.D. \\
\hline 3-metilpentano & 4,0 & 2,8 & 1,4 & N.D. & N.D. \\
\hline cis-2-penteno & 3,8 & 3,0 & N.D. & N.D. & N.D. \\
\hline metilciclopentano & 3,7 & 3,1 & N.D. & N.D. & N.D. \\
\hline $\begin{array}{l}\text { 1,2,4-trimetilben- } \\
\text { zeno }\end{array}$ & 3,0 & 2,3 & 7,9 & N.D. & N.D. \\
\hline $\begin{array}{l}\text { 1-etil,4-metilben- } \\
\text { zeno }\end{array}$ & 2,9 & 1,7 & N.D. & N.D. & N.D. \\
\hline 2-metilpentano & 2,7 & 1,4 & 7,8 & N.D. & N.D. \\
\hline benzeno & 2,7 & 1,1 & 6,6 & 1,1 & 5,17 \\
\hline trans-2-buteno & 2,7 & 1,9 & N.D. & N.D. & N.D. \\
\hline metilciclohexano & 2,6 & 1,4 & N.D. & N.D. & N.D. \\
\hline m,p-xileno & 2,6 & 1,9 & 16,1 & 10,4 & 4,2 \\
\hline etilbenzeno & 2,5 & 2,1 & 11,2 & 3,6 & 2,64 \\
\hline o-xileno & 2,4 & 1,5 & 8,2 & 3,0 & 2,83 \\
\hline n-octano & 2,4 & 2,2 & N.D. & N.D. & N.D. \\
\hline 3-metilhexano & 2,3 & 1,3 & 0,9 & N.D. & N.D. \\
\hline n-decano & 2,3 & 1,3 & N.D. & N.D. & N.D. \\
\hline n-nonano & 2,3 & 1,5 & 1,7 & N.D. & N.D. \\
\hline
\end{tabular}

* N.D. - Não Determinado; < L.D. - Menor que o Limite de Detecção. 
O valor médio para a concentração de COVs totais foi de $162,86 \mu \mathrm{g} \mathrm{m}^{-3}$, sendo que os $25 \mathrm{COVs}$ mais abundantes representam $86,7 \%$ da massa total dos compostos determinados. Estes valores são comparáveis ao determinado, no mesmo local, para 4 canisters, coletados às 9 horas, em março de 2004 (COVs totais $=220,5 \mu \mathrm{g}$ $\left.\mathrm{m}^{-3}\right) .{ }^{23}$ Nesse trabalho foram determinados $66 \mathrm{COVs}\left(\mathrm{C}_{3}-\mathrm{C}_{12}\right)$ através do método TO-14A, usando coleta instantânea. Os compostos aromáticos foram determinados, também, usando o método de cartuchos adsorvedores, extração com solvente e cromatografia gasosa, por Custodio et al. ${ }^{16}$ e por Martins et al. ${ }^{18}$ No primeiro caso foram coletadas 15 amostras, no período Janeiro a Agosto de 2008, e no segundo 30 amostras, entre Abril de 2012 e Fevereiro de 2013. Rigorosamente, os resultados não podem ser comparados em forma direta, já que os métodos de coleta e análise foram diferentes e as condições ambientais (principalmente as condições meteorológicas) também foram diferentes. Contudo, os resultados estão dentro da mesma ordem de grandeza e podem ser considerados satisfatórios.

As concentrações de benzeno determinadas neste trabalho se encontraram no intervalo 1,45 a 3,78 com um valor médio de $2,72 \mu \mathrm{g}$ $\mathrm{m}^{-3}$. Este valor é da mesma ordem de grandeza aos obtidos em $2012 \mathrm{e}$ $2013^{18}$ e mostrados na Tabela 4, porém, aproximadamente a metade dos obtidos anteriormente. O benzeno é considerado um composto carcinogênico, e é responsável por $68 \%$ do risco de câncer associado a poluentes veiculares. ${ }^{30}$ Ainda que no Brasil existam limites quanto às concentrações atmosféricas de benzeno, estas somente estão disponíveis para legislação trabalhista $\left(3,19-7,98 \mathrm{mg} \mathrm{m}^{-3}\right)$ que são referentes à riscos ocupacionais de exposição a este. No entanto, para limites de qualidade do ar, não existe nenhuma especificação para tal poluente. A União Européia ${ }^{31}$ estabelece na foma de Diretriz (2008/50/Ec), limite para a concentração ambiental de benzeno, associando este à proteção à saúde humana. Nesta, o limite é de $5,0 \mu \mathrm{g} \mathrm{m}^{-3}$, sendo este um valor máximo médio para o período de 1 ano. Já o Departamento de Meio Ambiente, Alimentos e Atividades Rurais do Reino Unido ${ }^{32}$ estabelece, para o mesmo período, um objetivo a ser alcançado como valor limite de benzeno, de 3,25 $\mu \mathrm{g} \mathrm{m}^{-3}$, para países como a Escócia e Irlanda, e um valor de 5,0 $\mathrm{g} \mathrm{m}^{-3}$, para a Inglaterra e País de Gales, em consonância com as recomendações da União Européia. Assim, os valores obtidos neste trabalho se encontram dentro dos limites estabelecidos pela União Européia.

A relação Benzeno/Tolueno determinada para estas amostras é de 0,36. Em geral, uma relação de aproximadamente 0,5 é considerada característica de emissões urbanas (fontes veiculares sem contribuições industriais). ${ }^{33}$

Quanto ao isopreno, considerado na literatura como o principal COV de origem biogênica, as concentrações individuais se encontram na faixa de 2,65 - 11,91 $\mu \mathrm{g} \mathrm{m}^{-3}$, com um valor médio de 4,34 $\mu \mathrm{g} \mathrm{m}^{-3}$. Para a cidade do Rio de Janeiro, conforme o nosso conhecimento, tal composto ainda não havia sido publicado, exceto por Martins et $a l .{ }^{22}$ que obtiveram, para diversas ruas da cidade, um valor médio de $1,39 \mu \mathrm{g} \mathrm{m}^{-3}$. Recentemente, Oliveira ${ }^{34}$ determinou em sua Tese de Doutorado valores médios de concentração de isopreno igual a 3,82 $\mu \mathrm{g} \mathrm{m}^{-3}$, para 7 canisters coletados na UERJ, campus Maracanã, e $4,53 \mu \mathrm{g} \mathrm{m}^{-3}$, para 14 canisters coletados na Floresta da Tijuca, (Casa do Pesquisador). As concentrações de isopreno encontradas neste trabalho são consideravelmente maiores que o valor determinado para Porto Alegre ${ }^{35}$ em 1996 - 1997, de $0,7 \mu \mathrm{g} \mathrm{m}^{-3}$, e em São Paulo ${ }^{36}$ em 2006 (1,5 a 2,3 $\mathrm{\mu g} \mathrm{m}^{-3}$ ), usando o método TO-14A. Ao comparar a relação média Benzeno/Isopreno determinada neste trabalho $(0,63)$ com os valores obtidos em Porto Alegre $(35,6)^{35}$ e São Paulo $(2,2)^{36}$ se observa que a contribuição relativa das emissões de origem biogênica na cidade de Rio de Janeiro é muito maior que em outras cidades do Brasil. A mesma conclusão é obtida ao comparar com valores obtidos recentemente em Bogotá, Colombia $(3,1)^{37}$ e na cidade de Pequim,
China, em $2006(2,36) \cdot{ }^{33}$ Os valores relativamente altos de isopreno na Praça Saens Peña podem ser explicados por esta ser uma região muito próxima à Mata Atlântica (Floresta da Tijuca e Morro de Sumaré), com abundante vegetação, e as amostras terem sido coletadas em dias ensolarados, que favorecem as emissões de isopreno e outros compostos de emitidos pelas plantas.

\section{CONCLUSÃO}

O método TO-15 se mostrou apropriado para a determinação de COVs em concentrações ambientais, sendo o LD nas faixas compreendidas entre 0,2 e $0,4 \mathrm{mg} \mathrm{m}^{-3}$ e 0,1 e $0,2 \mathrm{mg} \mathrm{m}^{-3}$ injetando um volume de amostra de $500 \mathrm{~mL}$ e $1 \mathrm{~L}$, respectivamente. Os resultados para os compostos de maior interesse neste trabalho, isopreno e compostos aromáticos, mostram que a mudança na concentração, geralmente com desvio negativo, é de aproximadamente $2-3 \%$ por dia, assim, as amostras deverão ser analisadas preferencialmente no prazo de até 8 dias de forma a garantir um erro menor que $25 \%$ preconizado pelo método. A repetitividade dos resultados, dentro dos critérios de qualidade estabelecidos pelo método, é atendida usando canisters de $6 \mathrm{~L}$ e injeções de $500 \mathrm{~mL}$. O uso de canisters de volume menor para amostras ambientais não se mostrou adequado porque não possibilita a realização de triplicatas. O uso de restritores de fluxo, para obtenção de uma amostra integrada, faz-se necessário para garantir a homogeneidade e reprodutibilidade dos resultados, condição que não é atingida com amostras instantâneas. Quando aplicado a amostras coletadas num ambiente urbano, o método se mostra satisfatório, fornecendo resultados compatíveis com valores da literatura.

\section{MATERIAL SUPLEMENTAR}

A Figura 1S (Cromatograma do branco), Figura 2S (Cromatograma dos compostos contidos no padrão Restek (57 COVs em nitrogênio), obtido em modo SCAN) e Figura 3S (Cromatograma de uma amostra típica coletada na Praça Afonso Viseu (Alto da Boa Vista, Rio de Janeiro) e a Tabela 1S (COVs analisados e íons usados para a quantificação) utilizados neste trabalho estão disponíveis em http:// quimicanova.sbq.org.br, na forma de arquivo PDF, com acesso livre.

\section{AGRADECIMENTOS}

Os autores agradecem o financiamento de FAPERJ (Processo E-26/110.440/2014), CNPq (Processo 490252/2011-7 e Processo 446158/2014-3) e Petrobras (Projeto 14281) e a colaboração do laboratório CENPES/PDEDS/AMA. ECCS e RLO agradecem ao CNPq e à CAPES, respectivamente, por bolsas de Doutorado. SMC e GA agradecem ao CNPq por bolsas de Produtividade.

\section{REFERÊNCIAS}

1. Hester, R. E.; Harrinson, R.; Volatile organic compounds in the atmosphere, The Royal Society of Chemistry: London, 1995.

2. Clean Air Act Amendments of 1990. htpp://www.epw.senate.gov/envlaws/cleanair.pdf. Acessada em fevereiro 2016.

3. U.S. Environmental Protection Agency; Compendium Method TO15, Determination of Volatile Organic Compounds (VOCs) in U.S. Air Collected in Specially-Prepared Canisters and Analyzed by Gas Chromatography-Mass Spectrometry (GC-MS), 1999. http://www.epa. gov/ttnamti1/files/ambient/airtox/to-15r.pdf, acessada em fevereiro 2016; U.S. Environmental Protection Agency. Compendium of Methods for the Determination of Toxic Organic Compounds in Ambient Air Second Edition, 1999. http://www.epa.gov/ttnamti1/files/ambient/airtox/ tocomp99.pdf, acessada em fevereiro de 2016. 
4. Coutant, R.W.; Theoretical Evaluation of Stability of Volatile Organic Chemicals and Polar Volatile Organic Chemicals in Canisters, U. S. Environmental Protection Agency, EPA Contract No. 68-DO0007, Work Assignment No. 45, Subtask 2, Battelle, Columbus, OH, June 1993. http://nepis.epa.gov/Exe/ZyPDF.cgi/30003VZY. PDF?Dockey=30003VZY.PDF. Acessada em fevereiro de 2016.

5. U.S. Environmental Protection Agency; Compendium of Methods for the Determination of Toxic Organic Compounds in Ambient Air. Second Edition. Compendium Method TO-14A. 1999. http://www.epa.gov/ ttnamti1/files/ambient/airtox/to-14ar.pdf, acessada em fevereiro 2016.

6. http://www.ipcc.ch/ipccreports/tar/wg1/140.htm, acessada em fevereiro de 2016.

7. Finalyson-Pitts, B. J.; Pitts Jr., J. N.; Chemistry of the upper and lower atmosphere: Theory, Experiments, and Applications. Academic Press: California, 2000.

8. Atkinson, R.; Atmos. Environ. 2000, 34, 2063.

9. Atkinson, R.; Arey, J.; Atmos. Environ. 2003, 37 (Spp. 2), S191.

10. Alves, C.; Pio, C.; Gomes, P.; Quim. Nova 2006, 29, 477.

11. http://cidades.ibge.gov.br/xtras/perfil.php?codmun=330455, acessada em fevereiro de 2016.

12. http://www.detran.rj.gov.br/_estatisticas.veiculos/07.asp, acessada em fevereiro de 2016.

13. http://www.parquedatijuca.com.br/files/guia_de_campo_PNT.pdf, acessada em fevereiro de 2015.

14. h t t p :// ww w.inea.rj.gov.br/Portal/ A g e ndas / BIODIVERSIDADEEAREASPROTEGIDAS/ UnidadesdeConservacao/ INEA_008594, acessada em fevereiro de 2015.

15. Azevedo, D. de A.; Moreira, L. S.; de Siqueira, D. S.; Atmos. Environ. 1999, 33, 4987.

16. Custodio, D.; Guimaraes, C. S.; Varandas, L.; Arbilla, G.; Chemosphere 2010, 79, 1064.

17. da Silva, C. M.; da Silva, L; L.; Correa, S. M. Arbilla, G.; Bull. Environ. Contam. Toxicol. submetido.

18. Martins, E. M.; Arbilla, G.; Bauerfeldt, G. F.; de Paula, M.; Chemosphere 2007, 67, 2096.

19. Fernandes, M. B.; Brickus, L.S.R.; Moreira, J. C.; Cardoso, J. N.; Chemosphere 2002, 47, 417.

20. Kelly, T. J.; Holdren, M. W.; Atmos. Environ. 1995, 29, 2595.

21. Corrêa, S. M.; Arbilla, G.; J. Braz. Chem. Soc. 2007, 18, 539.
22. Martins, E. M.; Nunes, A. C. L.; Correa, S. M.; J. Braz. Chem. Soc. 2015, 26, 197.

23. Martins, E. M.; Arbilla, G.; Gatti, L. V.; Bull. Environ. Contam. Toxicol. 2010, 84,175

24. Ribani, M.; Botolli, C. B. G.; Collins, C. H.; Sales, I. C.; Jardim, F.; Melo, L. F. C.; Quim. Nova 2004, 27, 771.

25. http://portal.anvisa.gov.br/wps/wcm/connect/4983b0004745975da005f 43fbc4c6735/RE_899_2003_Determina+a+publica\%C3\%A7\%C3\%A 3o+do+Guia+para+valida\%C3\%A7\%C3\%A3o+de+m\%C3\%A9todos +anal\%C3\%ADticos+e+bioanal\%C3\%ADticos.pdf?MOD=AJPERES, acessada em fevereiro de 2016.

26. Paschoal, J. A.; Rath, S.; Airoldi, F. P. Da S.; Reyes, F. G. R.; Quim. Nova 2008, 31, 1190.

27. Rappengluck, B.; Apel, E.; Bauerfeind, M.; Bottenheim, J.; Brickell, P.; Cavolka, P.; Ceche, J.; Gatti, L.; Hakola, H. , Honzak, J.; Junek, R.; Martin, D.; Noone, C.; Plass-Dulmer, Ch.; Travers, D.; Wang, D.; Atmos. Environ. 2006, 40, 7508.

28. Colón, M.; Pleila, J. D.; Hartlage, T. A.; Guardani, M. L.; Martins, M. H.; Atmos. Environ. 2001, 35, 4017.

29. Heo, G. S.; Lee, J. H.; Kim, S. W.; Lee, D. W.; Microchem. J. 2001, 70, 265.

30. Chan, C. Y.; Chan, L. Y.; Wang, X. M.; Liu, Y. M.; Lee, S. C.; Zou, S. C.; Sheng, G. Y.; Fu, J. M.; Atmos. Environ. 2002, 36, 2039.

31. European Union. Directive 2008/50/Ec of The European Parliament and of the Council of 21 May 2008 on Ambient Air Quality and Cleaner Air for Europe. Official Journal of the Europe, 2008.

32. DEFRA - Department for Environment, Food and Rural Affairs. The Air Quality Strategy for England, Scotland, Wales and Northern Ireland. Reino Unido, 2011, 2.

33. Duan, J.; Tan, J.; Yang, L.; Wu, S.; Hao, J.; Atmos. Res. 2008, 88, 25.

34. Oliveira, R. L.; Tese de Doutorado, Universidade Federal do Rio de Janeiro, Brasil, 2015.

35. Grosjean, E.; Rasmussen, R. A.; Grosjean, D.; Atmos. Environ. 1998, $32,3371$.

36. Alvim, D. S.; Gatti, L. V.; Correa, S. M.; Pretto, A.; Rossatti, C. de S.; Orlando, J. P.; Ciência \& Natura 2014, 36, 434.

37. Franco, J. F.; Pacheco, J.; Belalcázar, L. C.; Behrentz, E.; Atmósfera $\mathbf{2 0 1 5}, 28,1$ 